\title{
QUALIDADE DE VIDA EM PACIENTES CORONARIOPATAS
}

Maria Cecília Bueno Jayme Gallani* Roberta Cunha Rodrigues Colombo* Neusa Maria Costa Alexandre** Ana Maria Bressan-Biajone ${ }^{\star \star *}$

\section{Resumo}

Este estudo comparou as medidas de qualidade de vida (QV) entre 49 sujeitos com infarto do miocárdio (IM) e 27 com angina pectoris (AP), atendidos em ambulatório em um hospital universitário. Os dados foram obtidos por meio da aplicação de um questionário para levantamento de dados sociodemográficos e clínicos e do instrumento SF-36 para avaliação da QV. Constatou-se, em ambos os grupos comprometimento da QV nas dimensões: aspectos físicos, dor, estado geral de saúde, vitalidade, aspectos sociais e saúde mental Variáveis sociodemográficas e clínicas foram associadas com menores escores de QV em diferentes dimensões. Estudos futuros devem ser conduzidos, visando a ampliação do tamanho da amostra, e a compreensão da magnitude do efeito destas variáveis sobre a QV do coronariopata.

Descritores: qualidade de vida; coronariopatia; mortalidade

\begin{abstract}
This study compared measures of quality of life (QOL) in two groups of coronary heart disease patients: one with myocardial infarction (MI) and one with angina (A). The population studied consisted of 49 patients from Group MI and 27 patients from Group A. A questionnaire was used to identify sociodemographic and clinical data, as well as data from the SF-36 instrument, to assess the patients' QOL. It was observed in both groups that the QOL declined in the following aspects: physical functioning, pain, general health condition, vitality, social functioning, and mental health. Socio-demographic and clinical variables influenced the QOL score. Studies aiming at a larger number of individuals and a better understanding of the effects of the variables above upon the QOL of coronary patients are needed.
\end{abstract}

Descriptors: quality of life; coronary disease; mortality

Title: : Quality of life of coronary heart disease patients

\section{Resumen}

El objetivo de este estudio fue comparar las medidas de calidad de vida (CV) entre 49 sujetos con infarto de miocardio (IM) y 27 con angina pectoris $(A P)$, atendidos en unidad ambulatoria. Con aplicación de un cuestionario para el levantamiento de datos sociodemográficos y clínicos y del instrumento SF-36 para la evaluación de la CV, se observó, en ambos grupos, que se compromete la $\mathrm{CV}$ en los dominios: aspectos físicos, dolor, estado general de la salud, vitalidad, aspectos sociales y salud mental. Variables sociodemográficas y clínicas se asociaron con menores notas de CV en diferentes dominios. Se deberán conducir estudios futuros, buscando la comprensión de la magnitud del efecto de estas variables sobre la CV del coronariópata.

Descriptores: calidad de vida; coronariopatia; mortalidad

Título: Calidad de vida de sujetos con coronariopatia

\section{Introdução}

As doenças cardiovasculares (DCV) têm apresentado expressiva prevalência nos quadros de morbi-mortalidade na maioria dos países do ocidente, inclusive em países da América do Sul, como o Brasil. Dentre as DCV destaca-se a coronariopatia como grande causa de morte e incapacidade, na ocorrência de suas manifestações clínicas: infarto do miocárdio (IM), angina pectoris (AP) e a morte súbita.

Na literatura, há freqüentes registros sobre a influência da vivência da manifestação do evento isquêmico sobre a vida do sujeito. Tais repercussões não são restritas ao aspecto físico, mas englobam também os aspectos mental, social e a percepção de bem-estar geral ${ }^{(1,2)}$.

$\mathrm{Na}$ atualidade, além da mortalidade e da morbidade como avaliação de resultado do tratamento implementado para pacientes portadores de afecções crônicas, outra variável muito considerada tem sido a perspectiva de benefícios individuais, que se relacionam com a qualidade de vida (QV) ${ }^{(3,4)}$.

$O$ interesse de pesquisadores em transformar o conceito de qualidade de vida em uma medida quantitativa para uso em ensaios clínicos e em modelos econômicos é crescente para que esta medida possa ser comparada entre diversas populações e patologias ${ }^{(5)}$.

A importância da avaliação da QV junto a pacientes coronariopatas tem sido ratificada pela ampla utilização de diferentes instrumentos que permitam sua mensuração, nas diferentes áreas da saúde. Qualidade de vida é um conceito abstrato, altamente subjetivo, influenciado por valores pessoais e culturais, crenças, auto-conceitos, objetivos, idade e expectativa de vida ${ }^{(6)}$. Experiências vividas, como doenças, tratamentos, relações interpessoais e suporte social também exercem influência sobre a qualidade de vida. Os instrumentos que visam mensurar a QV procuram incluir questões que abordem as funções física, emocional e social, desempenho de papel, dor, sono e sintomas específicos de doença.

Considerando que a vivência do infarto e de angina podem ter repercussões diferentes na vida do sujeito, o presente estudo teve como objetivo comparar as medidas de QV entre dois grupos de pacientes coronariopatas: com antecedente de infarto do miocárdio (IM) e de angina pectoris (AP).

\section{Metodologia}

Tratou-se de um estudo não-experimental do tipo descritivo.

Foram sujeitos deste estudo pacientes coronariopatas, com história de manifestação clínica de IM e AP, atendidos no Ambulatório de Cardiologia, sub-especialidade de cardiopatia isquêmica, do Hospital de Clínicas da Universidade Estadual de Campinas (HC-UNICAMP), que atenderam aos seguintes critérios: capacidade de compreensão e de comunicar-se verbalmente e concordância em participar da pesquisa, oficializada através da assinatura do Termo de Consentimento Livre e Esclarecido (TCLE). Destaca-se que o padrão de atendimento não foi alterado para a realização do estudo.

\footnotetext{
* Enfermeira. Professor Doutor do Departamento de Enfermagem da Faculdade de Ciências Médicas (FCM) da Universidade Estadual de Campinas (UNICAMP).

** Enfermeira. Professor Livre Docente do Departamento de Enfermagem da FCM da UNICAMP.

*** Enfermeira com Aprimoramento em Enfermagem em Cuidados Intensivos.

E-mail do autor: rcolombo@fcm.unicamp.br
} 
Para a coleta de dados foi utilizado um questionário para o levantamento de dados sociodemográficos e dados relacionados à doença e tratamento - instrumento de caracterização, e o instrumento SF-36 (The Medical Outcomes Study 36-item Short-Form Health Survey) ${ }^{(7)}$, para avaliação da qualidade de vida.

O instrumento de caracterização teve como suporte teórico, outras investigações ${ }^{(8,9)}$ e foi divido em dois itens: dados sócio-demográficos (nome, número de registro no hospital [HC], idade, escolaridade, estado civil, com quem mora, procedência, profissão, vínculo empregatício e renda mensal); e dados relacionados à doença e ao tratamento (diagnóstico médico atual, data do diagnóstico, data do último episódio de IM, sintomas e tratamento).

Para obtenção de dados relacionados à qualidade de vida foi utilizado o SF 36, um indicador genérico da avaliação do estado de saúde, que pode ser utilizado em pesquisas, na prática clinica e em avaliações de programas de saúde. Tem sido empregado em estudos da população geral e em pacientes com diabetes, doenças pulmonares, problemas cardíacos entre outros ${ }^{(10,11)}$. Seu desenvolvimento teve como suporte teórico a definição multidimensional de saúde estabelecida pela Organização Mundial de Saúde abordando conceitos físicos e mentais. É constituído por escalas que avaliam oito dimensões: capacidade funcional; aspectos físicos; dor; aspectos sociais saúde mental; aspectos emocionais; vitalidade e aspecto geral de saúde ${ }^{(12)}$. A escolha desse questionário deve-se ao fato de já ter sido validado na cultura brasileira e de apresentar propriedades psicométricas confiáveis ${ }^{(5,12)}$.

Os dados coletados foram transportados para o software SAS - System for Windows, versão 6.12 para as seguintes análises: Descritiva, de Associação: teste Quiquadrado, de Comparação: teste de Mann-Whitney e Análise de Variância Multivariada (MANOVA). Foi adotado nível de significância de $5 \%$, ou seja, p-valor $£ 0,05$.

Ressalta-se que o estudo obteve parecer favorável do Comitê de Ética em Pesquisa da FCM-UNICAMP (Parecer $n^{\circ}$ 414/2002).

\section{Resultados}

\subsection{Dados gerais e sociodemográficos}

Os 76 pacientes estudados compuseram dois grupos: o grupo IM, de pacientes com infarto do miocárdio $(n=49)$ e o grupo AP, de pacientes com angina pectoris $(n=27)$.

Os pacientes estudados caracterizaram-se pelo predomínio do sexo masculino $(69,7 \%=53 / 76)$, idade compreendida entre 41 e 82 anos, com média de 60,4 $( \pm 9,2)$ anos, estado civil casado $(76,3 \%=58 / 76)$, morando com o cônjuge e/ou com os filhos $(90,8 \%=69 / 76)$. Metade do grupo estudado (38/76) referiu tempo de escolaridade menor ou igual a três anos; e a maioria $(84,2 \%=64 / 76)$ era procedente da cidade de Campinas ou região.

Quanto ao vínculo empregatício, mais da metade do grupo $(56,6 \%=43 / 76)$ era inativa (aposentadoria compulsória ou por invalidez, auxílio-doença ou desempregado), 27,6\% (21/ $76)$ eram ativos e $15,8 \%(12 / 76)$ do lar. Dos 43 sujeitos inativos, 30 eram do grupo IM $(61,2 \%=30 / 49)$ e 13 do grupo AP $(48,1 \%=13 / 27)$, embora esta diferença não tenha sido significativa estatisticamente. Foi verificado que o tipo de vínculo empregatício teve associação com outras variáveis, como: idade e anos de escolaridade, com maior concentração de sujeitos ativos no grupo com idade igual ou menor que 60 anos ( $p$-valor $=0,027)$ e com escolaridade superior a três anos de estudo ( $p$ valor $=0,029)$. Quanto à renda mensal familiar 60,0\% (45/76) referiram ser igual ou inferior a quatro salários-mínimos.

Em relação aos sintomas, foram relatados fadiga $(67,1 \%=51 /$ $76)$, angina $(40,8 \%=31 / 76)$, dispnéia $(43,4 \%=33 / 76)$, edema de membros inferiores $(27,3 \%=21 / 76)$ e arritmia $(26,3 \%=20 /$ 76).

Os grupos diferiram de maneira significativa no que se refere à escolaridade, tendo o grupo AP média de 6,1 $( \pm 5,3)$ anos de estudo e o grupo IM, média de $3,4( \pm 3,5)$ anos (p-valor $=0,032$ ), havendo também associação entre o grupo AP e o sintoma angina ( $63,0 \%$ dos pacientes do grupo AP e $28,6 \%$ dos pacientes do grupo IM relatavam apresentar periodicamente este sintoma ( $p$-valor $=0$, 0035).

\subsection{Qualidade de vida}

$\mathrm{Na}$ tabela 1 estão relacionados os escores de QV obtidos para o grupo como um todo e para cada um dos grupos IM e AP.

Os dados demonstram resultados semelhantes entre os dois grupos estudados nas oito dimensões que compõem o SF-36, o que foi confirmado por meio da aplicação do teste não-paramétrico de Mann-Whithney. Pode-se afirmar, entretanto, que, independentemente do tipo de manifestação clínica da coronariopatia, existe no grupo de estudo grande comprometimento da QV em seis das oito dimensões do SF36: aspectos físicos, dor, estado geral de saúde, vitalidade, aspectos sociais, e saúde mental, que apresentaram escores menores que a média do valor máximo que poderia ter sido obtido.

Para contextualizar os escores de QV obtidos neste estudo, procurou-se comparar seus resultados aos de outras pesquisas nacionais que empregaram o SF-36 (Tabela 2).

Como se observa na tabela 2 , existe uma variabilidade de escores de acordo com a situação clínica e cada uma das dimensões analisados do SF-36. É evidente, entretanto, que os sujeitos coronariopatas, em especial os deste estudo, exibiram a menor média de escores, em três das oito dimensões: vitalidade, aspectos sociais e saúde mental. Destaca-se que estes sujeitos apresentaram também escores baixos nas dimensões: aspectos físicos e dor.

Com o emprego do teste de Kruskal-Wallis foi notado que os sujeitos ativos obtiveram maior escore no domínio capacidade funcional do que os sujeitos inativos ou que eram do lar (médias respectivas: 73,2; 57,6 e 57,5; p-valor =0,043).

$\mathrm{Na}$ tabela 3 estão demonstradas as variáveis que se destacaram em cada domínio. Verificou-se que algumas variáveis determinaram diferenças nos escores obtidos. Destaca-se a presença de sintomas, como angina, arritmia e fadiga que influenciaram os escores de seis dimensões: capacidade funcional, dor, estado geral de saúde, vitalidade, aspectos sociais e aspectos emocionais. A variável sexo influenciou os escores dos dimensões: aspectos físicos, estado geral de saúde, e saúde mental. A variável renda familiar, por sua vez, destacou-se nas dimensões: vitalidade e saúde mental. O tempo decorrido desde o início da doença e o tipo de vínculo empregatício teve influência sobre apenas um domínio: capacidade funcional e saúde mental, respectivamente.

\section{Discussão}

Os dados aqui reportados não evidenciaram diferenças estatisticamente significativas nas medidas de QV nas oito dimensões do SF-36 entre os grupos estudados: IM e AP. Os escores observados, entretanto, reproduzem os achados da literatura relacionada à medida de $\mathrm{QV}$ em pacientes coronariopatas, mostrando baixa pontuação na maioria das 
Tabela 1- Resultado da média de escores obtidos em cada domínio do instrumento SF-36, de acordo com o conjunto de pacientes estudados e os grupos IM e AP. Campinas, 2001

\begin{tabular}{lll}
\hline \multicolumn{1}{c}{ Dimensões } & \multicolumn{1}{c}{ Variáveis destacadas } & p-valor \\
\hline Capacidade funcional & - Tempo decorrido desde o início da & 0,0550 \\
& doença & 0,0370 \\
& - Sintoma: angina & 0,0003 \\
Aspectos físicos & - Sintoma: arritmia & \\
Dor & - Sexo & 0,0340 \\
& - Sintoma: angina & 0,0053 \\
Estado Geral de Saúde & - Sintoma: arritmia & 0,0063 \\
Vitalidade & - Sexo & 0,0325 \\
& - Sintoma: fadiga & 0,0358 \\
Aspectos Sociais & - Renda familiar & 0,0336 \\
Aspectos Emocionais & - Sintoma: fadiga & 0,0383 \\
Saúde Mental & - Sintoma: fadiga & 0,0075 \\
& - Sintoma: arritmia & 0,0120 \\
& - Sexo & $<0,0001$ \\
& -Vínculo empregatício & 0,0333 \\
& - Renda familiar & 0,0010 \\
\hline
\end{tabular}

Tabela 2 - Resultado da média de escores obtidos em cada domínio do instrumento SF-36, em diferentes grupo de pacientes. Campinas, 2001

\begin{tabular}{lcccc}
\hline \multicolumn{1}{c}{ Escalas } & $\begin{array}{c}\text { Lombalgia } \\
\text { crônica }^{(13,15)}\end{array}$ & $\begin{array}{c}\text { Pacientes após 6 } \\
\text { meses de } \\
\text { hemodiálise }^{(14)}\end{array}$ & $\begin{array}{c}\text { Angina } \\
\text { pectoris }^{(13)}\end{array}$ & $\begin{array}{c}\text { Pacientes }^{(\mathrm{a})} \\
\text { coronariopatas }^{(\mathrm{a})}\end{array}$ \\
\hline Capacidade funcional & 84,6 & 53,0 & 23,8 & 61,9 \\
Aspectos físicos & 79,0 & 43,0 & 42,6 & 49,4 \\
Dor & 37,4 & 68,0 & 45,6 & 49,4 \\
Estado Geral de Saúde & 51,2 & 61,0 & 41,5 & 44,8 \\
Vitalidade & 49,2 & 50,0 & 40,3 & 40,0 \\
Aspectos Sociais & 48,0 & 66,0 & 56,9 & 45,9 \\
Aspectos Emocionais & 74,2 & 56,0 & 45,5 & 61,7 \\
Saúde Mental & 58,6 & 60,0 & 61,2 & 47,3 \\
\hline
\end{tabular}

Tabela 3 - Distribuição das variáveis destacadas em cada uma das dimensões do instrumento SF-36, por meio da MANOVA. Campinas, 2001

\begin{tabular}{lccc}
\hline \multicolumn{1}{c}{ Escalas } & $\begin{array}{c}\text { Conjunto (IM+AP) } \\
\mathrm{n}=76\end{array}$ & $\begin{array}{c}\text { Grupo IM } \\
\mathrm{n}=49\end{array}$ & $\begin{array}{c}\text { Grupo AP } \\
\mathrm{N}=27\end{array}$ \\
\hline Capacidade funcional & $61,9( \pm 26,3)$ & $61,4( \pm 26,4)$ & $62,8( \pm 26,7)$ \\
Aspectos físicos & $49,4( \pm 41,0)$ & $46,9( \pm 40,7)$ & $53,7( \pm 42,0)$ \\
Dor & $36,2( \pm 12,1)$ & $37,5( \pm 11,1)$ & $33,9( \pm 13,6)$ \\
Estado Geral de Saúde & $44,8( \pm 12,6)$ & $45,6( \pm 11,6)$ & $43,5( \pm 14,1)$ \\
Vitalidade & $40,0( \pm 14,6)$ & $41,7( \pm 14,2)$ & $36,8( \pm 15,0)$ \\
Aspectos Sociais & $45,9( \pm 13,4)$ & $47,2( \pm 13,8)$ & $43,5( \pm 12,7)$ \\
Aspectos Emocionais & $61,8( \pm 44,8)$ & $61,9( \pm 45,1)$ & $61,7( \pm 45,0)$ \\
Saúde Mental & $47,3( \pm 14,5)$ & $48,1( \pm 13,9)$ & $45,9( \pm 15,7)$ \\
\hline
\end{tabular}

dimensões avaliadas. Observa-se que, quando os escores obtidos neste estudo, são comparados a escores de pesquisas realizadas com outras condições clínicas, como lombalgia e realização crônica de hemodiálise, as dimensões: aspectos físicos, dor, estado geral de saúde, vitalidade, aspectos sociais e saúde mental encontram-se, expressivamente, comprometidas.

A análise mais detalhada dos escores obtidos, em conjunto com as variáveis sociodemográficas e clínicas também pesquisadas, mostra que, neste grupo de pacientes, se o tipo de manifestação clínica da doença não foi correlacionado com diferenças na medida de QV, outros fatores mostraram-se importantes como influenciadores da QV, sendo eles: sexo, tipo de vínculo empregatício, tempo decorrido desde a manifestação da doença e a presença de sintomas como angina, arritmia e fadiga.

Em relação ao sexo, observou-se menor pontuação para mulheres, quando comparadas aos homens, em algumas dimensões. $\mathrm{Na}$ atualidade, a coronariopatia é causa significativa de mortalidade entre as mulheres. No Brasil, segundo informações do Datasus(16), em 1998 as DCV foram responsáveis por aproximadamente $31,3 \%$ de todas as causas de mortalidade para as mulheres, sendo a coronariopatia, o diagnóstico efetuado em aproximadamente $8,0 \%$ de todas as causas.

Todavia, é curioso notar os registros crescentes na literatura sobre as diferenças existentes na abordagem da mulher no diagnóstico e tratamento da coronariopatia. É 
observado que, desde a investigação da doença, de suas manifestações, passando pelo tipo de abordagem terapêutica, até os processos de reabilitação, a mulher encontra-se, sempre, em desvantagem em relação ao homem, sendo arrolada com menor freqüência a programas de reabilitação ou recebendo menor proporção de terapêuticas medicamentosas I invasivas agressivas quando comparada aos pacientes do sexo masculino ${ }^{(17)}$, condições que podem comprometer de maneira significativa o prognóstico clínico e de readaptação psicossocial.

Em relação ao tipo de vínculo empregatício, os dados mostraram que os sujeitos ativos apresentaram melhor pontuação que os inativos. Trata-se de achado significativo, visto que da totalidade dos sujeitos entrevistados, $71,1 \%$ referiram alguma modificação na atividade de trabalho exercida, em decorrência da manifestação da doença. Mais da metade do grupo $(56,6 \%=43 / 76)$ era inativa, sendo que destes, $44,7 \%$ atribuíram a inatividade à doença. Estes achados reproduzem o que a literatura tem trazido sobre o impacto da coronariopatia na sociedade - que além de ser uma doença com formas graves de manifestação clínica, retira precocemente o sujeito da vida produtiva, com conseqüências negativas para ele e para a sociedade ${ }^{(18)}$. Uma das conseqüências negativas dessa ocorrência pode ser evidenciada neste estudo, ao ser constatada menor pontuação de medida de QV entre os sujeitos inativos.

O tempo decorrido desde a manifestação da doença foi associado de maneira negativa ao domínio capacidade funcional, de maneira que os sujeitos com maior tempo de manifestação apresentaram menor escore neste domínio. Seria interessante buscar investigar, em estudos futuros, se existe a reprodução desse achado com ampliação do tamanho da amostra, e quais seriam os mecanismos pelos quais isso ocorre.

A presença dos sintomas: angina, arritmia e fadiga também influenciaram as medidas de QV na maioria das dimensões. Em estudo prévio observou-se que os sujeitos com história de IM retratam tal vivência como um grande sofrimento, assim como o medo de sua reincidência ${ }^{(18)}$. Parece possível afirmar, que os sintomas, além de limitarem as atividades cotidianas dos sujeitos, possam, também, evocar a possibilidade da manifestação de um novo evento isquêmico, daí sua influência sobre as dimensões: capacidade funcional, dor, vitalidade, aspectos sociais e aspectos emocionais.

Estes achados reiteram a necessidade de serem realizados esforços para que as intervenções junto ao paciente coronariopata levem em consideração não apenas a efetividade do tratamento em relação ao aumento da sobrevida, mas a qualidade com que o sujeito desfruta esta sobrevivência. Estudos futuros devem ser conduzidos, visando a ampliação do tamanho da amostra, e a compreensão da magnitude do efeito das variáveis destacadas acima sobre a QV do sujeito coronariopata. Estudos Iongitudinais também devem ser encorajados, para avaliar se intervenções voltadas especificamente para abordagem destas variáveis, realmente resultam na melhora da QV destes sujeitos. O emprego associado de instrumentos de medida de QV doençaespecíficos também deve ser considerado, uma vez que apresentam sensibilidade clínica e maior poder de detecção de mudanças na QV em resposta a intervenções clínicas específicas, obtendo-se assim, a avaliação genérica e específica da QV dos sujeitos estudados.

\section{Conclusão}

Os dados do presente estudo permitiram concluir que não houve diferença estatisticamente significativa entre as medidas de QV, realizadas por meio do instrumento genérico
SF-36, para os grupos IM e AP estudados. Entretanto, as variáveis: sexo, tipo de vínculo empregatício, tempo decorrido desde a manifestação da doença e a presença dos sintomas (angina, arritmia e fadiga) foram associadas com menor pontuação nas medidas de QV em diferentes dimensões.

Estudos futuros devem ser conduzidos, visando a ampliação do tamanho da amostra, e a compreensão da magnitude do efeito das variáveis destacadas sobre a QV do sujeito coronariopata.

\section{Referências}

1. Lukkarinen $\mathrm{H}$. Quality of life in coronary artery disease. Nursing Research, 1998; 47(6):337-43.

2. Thompson DR, Roebuck A. The measurement of health-related quality of life in patients with coronary heart disease. Journal of Cardiovascular Nursing, 2001;16(1):28-33.

3. Rukholm E, Mcgirr M.A quality-of-life index for clients with ischemic heart disease: establishing reliability and validity. Rehabilitation Nursing, 1994; 19(1):12-6.

4. Silva DMGV, Vieira RM, Koshnik Z, Azevedo M, Soares SS. Qualidade de vida de pessoas com insuficiência renal crônica em tratamento hemodialítico. Revista Brasileira de Enfermagem, 2002; set/out 55(5): $562-7$.

5. Ciconelli RM, Ferraz MB, Santos W, Meinão I, Quaresma MR. Tradução para o português e validação do questionário genérico de avaliação de qualidade de vida "Medical Outcomes Study 36-Item Short-Form Health Survey (SF-36)". Revista Brasileira de Reumatologia, 1999; 39(3): 143-50.

6. Kelleher CJ, Cardozo LD, Khullar V. A new questionnaire to assess the quality of life of urinary incontinent women. Bristh Journal of Obstetrics and Gynecology, 1997;104:1374-9.

7. Ware JE, Sherbourne CD. The MOS 36-Item Short-Form Health Survey (SF 36). Medical Care, 1992; 30(6): 473-83.

8. Colombo RCR. Reabilitação no infarto do miocárdio: análise dos comportamentos relacionados a fatores de risco para coronariopatia [tese de Doutorado em Enfermagem]. Ribeirão Preto (SP): Escola de Enfermagem de Ribeirão Preto, Universidade de São Paulo; 2000. $319 f$.

9. Gallani MCBJ. O exercício físico e o paciente infartado: determinantes comportamentais. São Paulo [tese de Doutorado em Enfermagem]. São Paulo: Escola de Enfermagem, Universidade de São Paulo; 2000. $212 f$.

10. Hutchinson A, Bentzen N, Konig-Zahn C. Cross cultural health outcome assessment: a user's guide. The Netherlands: Ergho;1996.

11. Patrick DL et al. Assessing health-related quality of life in patients with sciatica. Spine, 1995; 20(27):1899-909.

12. Mcdowell I, Newell C. Measuring health: a guide to rating scales and questionnaires. New York: Oxford University Press; 1996. p. 44656.

13. Gurgueira GP, Alexandre NMC. Qualidade de vida em trabalhadoras de enfermagem com dor lombar crônica. Relatório Final, São Paulo: FAPESP; 2001.

14. Dougherty $\mathrm{CM}$ et al. Comparison of three quality of life instruments in stable angina pectoris Seattle Angina Questionnaire, Short Form Health Survey (SF-36) and Quality of Life Index-Cardiac Version III. Journal of Clinical Epidemiology, 1998; 51:560-77.

15. Rodrigues-Neto JF. Qualidade de vida em pacientes com hemodiálise: um estudo prospectivo. [tese de Doutorado]. São Paulo: Escola Paulista de Medicina, Universidade Federal de São Paulo, 2001. 108f.

16. Ministério da Saúde (BR). DATA SUS. Dados de mortalidade de 1998. Brasília (DF); 2000. Disponível em <http://www.datasus.gov.br/ principal.htm>. Acessado em: 28 out 2000.

17. Halm M. Women and cardiac rehabilitation: referral and compliance patterns. Journal of Cardiovascular Nursing, 1999;13(3): 83-92.

18. Gillette W. Comité report on economic, administrative and legal factors influencing the insurability and employability of patients with ischemic heart disease. Journal of American College of Cardiology, 1993; 14:1010.

Data de recebimento: 20/02/2003

Data de aprovação: 26/06/2003 